\title{
Terapêutica das coagulopatias em pacientes atendidos no hemocentro de uma região
}

\section{do nordeste brasileiro}

\section{Therapeutics of coagulopathies in patients attended at the blood center of a northeastern region of}

Brazil

Terapéutica de las coagulopatías en pacientes atendidos en el centro de sangre de una región nororiental de Brasil

Weber de Santana Teles ORCID: https://orcid.org/0000-0003-1770-8278 Centro de Hemoterapia de Sergipe, Brasil

E-mail: arteecura@hormail.com

Max Cruz da Silva

ORCID: https://orcid.org/0000-0002-6944-5986

Faculdade Pio Décimo, Brasil

E-mail: maxlfi@hotmail.com

Ruth Cristini Torres

ORCID: https://orcid.org/0000-0002-8664-192X Instituto de Hematologia e Hemoterapia de Sergipe, Brasil

E-mail: ruthcristini@gmail.com

Marcel Vinícius Cunha Azevedo

ORCID: https://orcid.org/0000-0002-5312-3333

Centro Universitário Estácio Sergipe, Brasil

E-mail: marcelvinicius49@gmail.com

Ana Fatima Souza Melo de Andrade

ORCID: https://orcid.org/0000-0002-7024-6175

Centro Universitário Estácio de Sergipe, Brasil

E-mail: anafatimamelo@hotmail.com

Taíssa Alice Soleade Calasans

ORCID: https://orcid.org/0000-0003-0460-4437

Universidade Tiradentes, Brasil

E-mail: taissa.asc@gmail.com

Alejandra Debbo

ORCID: https://orcid.org/0000-0002-7743-5921

Universidade Federal de Sergipe, Brasil

E-mail: aledebbo@hotmail.com

Ângela Maria Melo Sá Barros

ORCID: https://orcid.org/0000-0003-4087-3247

Universidade Federal do Rio de Janeiro, Brasil

E-mail: angelsamelo@hotmail.com

Paulo Celso Curvelo Santos Junior

ORCID: https://orcid.org/0000-0001-5834-6782 Universidade Tiradentes, Brasil

E-mail: paulo.curvelo.jr@gmail.com

Rute Nascimento da Silva

ORCID: https://orcid.org/0000-0002-2719-1623

Universidade Tiradentes, Brasil

E-mail: silva_rute@hotmail.com

\section{Resumo}

A hemofilia é uma doença hemorrágica congênita e genética que se configura pela deficiência de uma proteína plasmática responsável pela coagulação, e acomete mais indivíduos do sexo masculino devido a uma alteração no cromossomo X. Os dados foram analisados no hemocentro de uma região do nordeste brasileiro. O objetivo do trabalho foi avaliar o perfil epidemiológico e tratamento das coagulopatias em pacientes atendidos no Hemocentro, durante os anos de 2018 e 2019. No total foram analisados 242 prontuários de pacientes, e tabulados em frequência absoluta utilizando variáveis epidemiológicas, tipo de hemofilia, e uso dos fatores de coagulação. Os resultados mostraram que dos 242 pacientes portadores de coagulopatias, 154 (63,6\%) eram sexo masculino e 87(36,4\%), destes, $113(47 \%)$ apresentaram hemofilia A, com maior prevalência entre 16 à 30 (43\%) anos. A hemofilia B $18(8 \%)$ pacientes, com 
faixa etária entre 36 a 50 (33\%) anos. Em relação a localização a região urbana apresentou a maior prevalência para os portadores de hemofilia do tipo A. Dos 141pacientes que receberam fator de coagulação, 76 fizeram o uso de o Fator VIII recombinante, 66 fizeram o uso da Dose Domiciliar de Urgência (D.D.u). A quantidade de Unidades Internacionais (UI) dispensadas com maior proporção ocorreu no mês de agosto de 2018 e junho de 2019. Conclui-se que a necessidade de implementação de acompanhamento aos pacientes que fazem uso dos fatores de coagulação, assim como a implementação de investimentos do governo federal afim de que o tratamento tenha continuidade.

Palavras-chave: Hemofilia; Diagnóstico; Condutas terapêuticas.

\begin{abstract}
Hemophilia is a congenital and genetic hemorrhagic disease that is characterized by a deficiency of a plasma protein responsible for clotting, and affects more males due to an alteration in the $\mathrm{X}$ chromosome. Data were analyzed at the blood center of a region in northeastern Brazil. The objective of this study was to evaluate the epidemiological profile and treatment of coagulopathies in patients treated at the Blood Center during the years 2018 and 2019. In total, 242 patient records were analyzed, and tabulated in absolute frequency using epidemiological variables, type of hemophilia, and use of coagulation factors. The results showed that of the 242 patients with coagulopathies, $154(63.6 \%)$ were male and $87(36.4 \%)$, of these, $113(47 \%)$ had hemophilia A, with a higher prevalence between 16 and 30 (43\%) years old. Hemophilia B 18 (8\%) patients, aged between 36 and 50 (33\%) years. Regarding the location, the urban region had the highest prevalence for patients with hemophilia type A. Of the 141 patients who received coagulation factor, 76 used recombinant Factor VIII, 66 used Urgent Home Dose (DDU). The number of International Units (UI) dispensed with the highest proportion occurred in August 2018 and June 2019. It is concluded that the need to implement monitoring of patients who make use of coagulation factors, as well as the implementation of investments the federal government in order for the treatment to be continued.
\end{abstract}

Keywords: Hemophilia; Diagnosis; Therapeutic conducts.

\title{
Resumen
}

La hemofilia es una enfermedad hemorrágica congénita y genética que se caracteriza por la deficiencia de una proteína plasmática responsable de la coagulación, y afecta a más hombres debido a una alteración en el cromosoma X. Los datos fueron analizados en el centro de sangre de una región del noreste de Brasil. El objetivo de este estudio fue evaluar el perfil epidemiológico y tratamiento de las coagulopatías en pacientes atendidos en el Centro de Sangre durante los años 2018 y 2019. En total, se analizaron 242 historias clínicas y se tabularon en frecuencia absoluta utilizando variables epidemiológicas, tipo de hemofilia. y uso de factores de coagulación. Los resultados mostraron que de los 242 pacientes con coagulopatías, 154 (63,6\%) eran del sexo masculino y 87 (36,4\%), de estos $113(47 \%)$ tenían hemofilia A, con una mayor prevalencia entre los 16 y 30 (43\%) años. viejo. Pacientes con hemofilia B 18 (8\%), con edades comprendidas entre 36 y 50 (33\%) años. En cuanto a la ubicación, la región urbana tuvo la mayor prevalencia de pacientes con hemofilia tipo A. De los 141 pacientes que recibieron factor de coagulación, 76 usaron Factor VIII recombinante, 66 usaron Urgent Home Dose (DDU). El número de Unidades Internacionales (UI) prescindidas de mayor proporción ocurrió en agosto de 2018 y junio de 2019. Se concluye que la necesidad de implementar el monitoreo de los pacientes que hacen uso de factores de coagulación, así como la implementación de inversiones del gobierno federal en Orden de continuación del tratamiento.

Palabras clave: Hemofilia; Diagnóstico; Conductas terapéuticas.

\section{Introdução}

A hemofilia é uma doença hemorrágica congênita e genética que se configura pela deficiência de uma proteína plasmática responsável pela coagulação. Diversos elementos do sangue possuem funções específicas, nas quais estão os fatores de coagulação, proteínas que intervém no estancamento das hemorragias. Cada tipo de coagulopatia é caracterizada pela deficiência de um fator, sendo que a hemofilia A o fator VIII é o que se encontra com deficiência, e na hemofilia B o fator IX (Croteau, 2018).

De acordo com as informações da Federação Mundial de Hemofilia (2017) cerca de 196 mil pessoas são portadoras do distúrbio em que o sangue não coagula normalmente classificada como hemofilia, sendo a Índia o local de maior incidência da doença, seguido dos Estados Unidos e China. O Brasil encontra-se na quarta colocação com aproximadamente 12.400 indivíduos acometidos pela hemofilia. 
As hemofilias podem ser caracterizadas através dos níveis plasmáticos do fator deficiente, sendo classificadas como grave quando o nível de fator circulante é menor que 1\%; moderada quando está entre $1 \%$ e $5 \%$ e leve se superior a 5\%, comparadas ao nível normal que é de 100\%, ou seja, o mesmo que uma unidade de fator por mililitro de sangue. (Evatt, 2005).

As manifestações clínicas das coagulopatias são os sangramentos que ocorrem de forma espontânea ou induzida por traumas ou até mesmo por cirurgia. (Pio et al, 2009). Os problemas que geram maiores complicações para pacientes com hemofilia competem ao desenvolvimento de inibidores, que são anticorpos policlonais da classe IgG direcionados contra os fatores VIII ou IX infundidos (aloanticorpos). Sendo assim, os pacientes enfermos passam a não responder à infusão do fator deficiente e apresentam episódios hemorrágicos de difícil controle. (Brasil, Ministério Da Saúde, 2014).

Além destas manifestações clínicas, as coagulopatias trazem consequências em diversos cenários da particularidade dos pacientes, como convívio na sociedade, relacionamentos, na educação e no ambiente de trabalho.

Neves, et al. (2021) verificou que há diferenças na qualidade de vida dos pacientes portadores de coagulopatias em relação aos indivíduos sem a doença no que se refere as condições de trabalho e falhas na isonomia salarial.

São vários os tipos de coagulopatias, e para uma orientação terapêutica adequada, é indispensável um diagnóstico diferencial entre essas doenças, que é realizado por meio da história clínica e de testes laboratoriais. Os diagnósticos laboratoriais dos pacientes hemofílicos são estabelecidos pelo Tempo de Protrombina (TP), Tempo de Tromboplastina Parcial ativado (TTPa), tempo de sangramento, contagem de plaquetas, (Silva et al, 2006). As dosagens específicas são baseadas na quantificação da atividade coagulante dos fatores VIII (FVIII:C) e IX (FIX:C) pela técnica coagulométrico e quantificação do FVIII:C, pelo método cromogênico (Rodrigues, et al., 2018).

Dentre as coagulopatias hereditárias, as hemofilias A B e a doença Von Willebrand (DVW) são mais comuns, podendo atingir cerca de $97 \%$ dos indivíduos com alguma deficiência na hemostasia. E as deficiências de fatores I, II, V, VII, X e XIII são consideradas raras. (Peyvandi, et al., 2006).

O pilar do tratamento das coagulopatias hemofílicas é a infusão do concentrado de fator deficiente, que pode ser de origem plasmática ou recombinante. Existem dois tipos de tratamento para reposição: o de demanda e o profilático (Alcântara, et al., 2019).

O tratamento de demanda é àquele que ocorre após um episódio de sangramento e o tratamento profilático se refere àquele que ocorre anteriormente ao desenvolvimento de um episódio de hemorragia, precavendo que ocorra futuros danos nas articulações proporcionando aos pacientes uma vida mais ativa. (Vandenberg, et al., 2015)

O tratamento profilático com reposição constante na quantidade de fator VIII e IX circulante, é suficiente para evitar o surgimento de sangramentos. Antigamente se adotava o tratamento sob demanda, onde os fatores eram administrados apenas quando havia sangramentos, ocasionando complicações articulares (Brasil, 2018).

Baseado nisto, o presente trabalho possui como objetivos avaliar o perfil epidemiológico e tratamento das coagulopatias em pacientes atendidos no Hemocentro de uma região do Nordeste brasileiro durante o período de 2018 a 2019.

\section{Metodologia}

A pesquisa foi realizada no hemocentro de uma região do nordeste brasileiro, trata-se de uma análise retrospectiva de dados de 242 pacientes que necessitaram de administração de fator (VIII e IX) atendidos pelo hemocentro. Foram utilizados os prontuários dos pacientes portadores de hemofilia, sendo avaliado variáveis epidemiológicas tais como: gênero, idade, localização, tipo de hemofilia, pacientes que fizeram o uso de fatores: VIII, VIII R, VIII Y, IX e FEIBA e Dose Domiciliar de Urgência (D.D.u) e da quantidade de Unidades Internacionais (UI) dispensados entre janeiro de 2018 a dezembro de 2019. Os dados foram organizados e analisados no Microsoft Excel 2016 e demonstrados em tabelas e gráficos. 


\section{Resultados}

Foram pesquisados 242 pacientes portadores de coagulopatias atendidos no Hemocentro de uma região do nordeste brasileiro, durante o período de 2018 a 2019, sendo 154 do sexo masculino (63,6\%) e 87 do sexo feminino (36,4\%), (Gráfico 1).

Gráfico 1. Frequência de coagulopatias dos pacientes atendido no Hemocentro de uma região do nordeste Brasileiro no período de janeiro de 2018 a dezembro 2019.

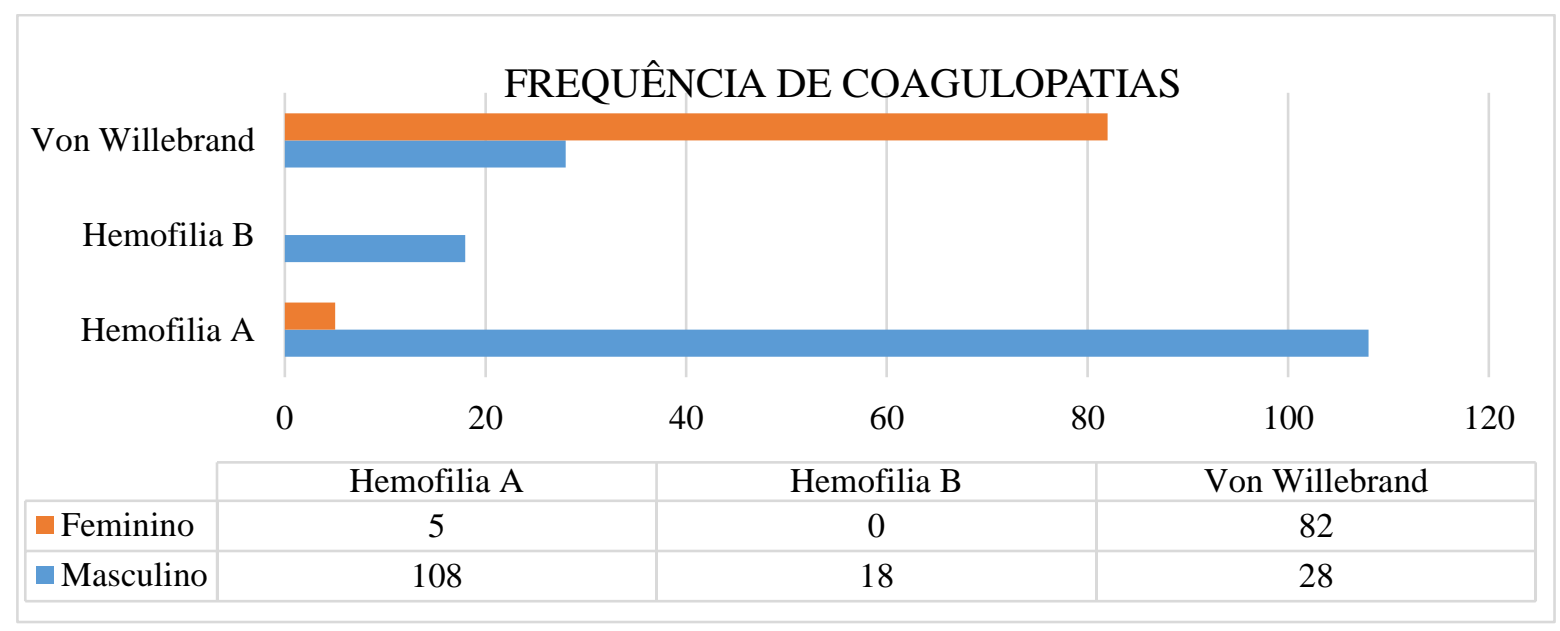

Fonte: Elaboração própria.

Destes $113(47 \%)$ apresentaram hemofilia A, com maior prevalência de (43\%) entre 16 à 30 anos. Em relação à hemofilia B 18 (8\%) com faixa etária entre 36 a 50 anos (33\%). Quanto à doença de Von Willebrand, apresentam 110 casos $(45,4 \%)$, sendo $82(74,55 \%)$ casos do sexo feminino e do sexo masculino 28 (25,45\%). (Tabela 1$)$.

Tabela 1. Classificação da faixa etária dos pacientes com Hemofilia A e B tratada no Hemocentro de uma região do nordeste brasileiro.

\begin{tabular}{|c|c|c|c|c|}
\hline \multirow{2}{*}{$\begin{array}{l}\text { FAIXA ETÁRIA } \\
\text { 02-15 anos }\end{array}$} & \multicolumn{2}{|c|}{ HEMOFILIA A \% } & \multicolumn{2}{|c|}{ HEMOFILIA B \% } \\
\hline & 4 & $30 \%$ & 3 & $16 \%$ \\
\hline 16-30 anos & 49 & $43 \%$ & 4 & $21 \%$ \\
\hline 31-45 anos & 18 & $16 \%$ & 6 & $33 \%$ \\
\hline 46-60 anos & 10 & $9 \%$ & 3 & $16 \%$ \\
\hline$\geq 65$ anos & 1 & $1 \%$ & 2 & $16 \%$ \\
\hline Sem classificação & 1 & $1 \%$ & - & \\
\hline Total & 113 & $100 \%$ & 18 & $100 \%$ \\
\hline
\end{tabular}

Fonte: Elaboração própria.

De acordo com a pesquisa desenvolvida 44 (39\%) dos portadores de Hemofilia A residem na área urbana de uma região do nordeste brasileiro, e os demais pacientes residem em outras cidades 36 (31\%). Em relação a hemofilia B a maior prevalência foi na zona urbana com 3 (16\%). Dos 141 pacientes que tiveram a dispensação ou administração de fator de coagulação, 76 fizeram o uso de o Fator VIII (recombinante), caracterizando a maior prevalência. E 62 pacientes fazem ou fizeram o uso de 
Dose Domiciliar de Urgência (D.D.u). De acordo com a REVISTA FATOR VIDA (2014) o fator recombinante utilizado é produzido em laboratórios, por tecnologia de ácido nucléico recombinante, dentro de células de ovários de animais, onde essa célula passa por uma etapa de recombinação de DNA e recebe o gene responsável pela produção da proteína do fator VIII tornando-se assim fator recombinante. (Tabela 2).

Tabela 1. Classificação dos pacientes que tiveram acesso a dispensação de fatores de coagulação e Dose Domiciliar de urgência (D.D.u) no Hemocentro coordenador do Estado de Sergipe.

\begin{tabular}{cccccccc}
\hline Fator & Fator VIII & Fator VIIIr & Fator VIIIy & Fator IX & FEIBA & D.D.U \\
\hline Pacientes & 28 & $\mathbf{7 6}$ & 43 & 7 & 8 & 62 \\
\hline Total & 28 & 76 & 43 & 7 & 8 & 62 \\
\hline
\end{tabular}

Fonte: Elaboração própria.

Através dos dados obtidos na presente pesquisa, foi possível totalizar a quantidade de Unidades Internacionais (UI) referentes aos fatores: VIII, VIII R, VIII Y, IX e FEIBA; que foram dispensados durante o período de janeiro de 2018 a dezembro de 2019. Segundo os dados, a maior prevalência de dispensação de UI, ocorreu no mês de agosto de 2018 (570.300 UI) e junho de 2019 (769.500 UI). No ano de 2018 foram dispensados 6.067.750 UI, e em 2016 (6.872.750).

Tabela 2. Classificação quantitativa da (UI) dispensado entre janeiro de 2018 a dezembro de 2019 no Hemocentro de uma região do nordeste brasileiro.

\begin{tabular}{cccc}
\hline Ano de 2015 & Unidades internacionais (UI) & Ano de 2016 & Unidades internacionais (UI) \\
\hline Janeiro & 485.500 & Janeiro & 553.500 \\
\hline Fevereiro & 491.000 & Fevereiro & 576.750 \\
\hline Março & 566.000 & Março & 464.000 \\
\hline Abril & 517.500 & Abril & 593.300 \\
\hline Maio & 531.250 & Maio & 435.750 \\
\hline Junho & 411.250 & Junho & $\mathbf{7 6 9 . 5 0 0}$ \\
\hline Julho & 498.500 & Julho & 471.200 \\
\hline Agosto & $\mathbf{5 7 0 . 3 0 0}$ & Agosto & 647.750 \\
\hline Setembro & 551.950 & Setembro & 496.000 \\
\hline Outubro & 496.250 & Outubro & 526.500 \\
\hline Novembro & 451.250 & Novembro & 673.750 \\
\hline Dezembro & 497.500 & Dezembro & 664.750 \\
\hline Total & $\mathbf{6 . 0 6 7 . 7 5 0}$ & Total & $\mathbf{6 . 8 7 2 . 7 5 0}$ \\
\hline
\end{tabular}

Fonte: Elaboração própria.

\section{Discussão}

Segundo o Manual de reabilitação na hemofilia do Ministério da Saúde, (2015), a prevalência das coagulopatias e demais transtornos hemorrágicos no Brasil, observou-se os diversos tipos de coagulopatias hereditárias, 14.863 (70,55\%) corresponde aos pacientes que são do sexo masculino e $6.203(29,45 \%)$ do sexo feminino, totalizando 21.066 casos.

No presente estudo foi observado que a maior prevalência da hemofilia A, acomete indivíduos do sexo masculino, que de acordo com Pasquini et al (2004), a doença afeta com maior frequência os homens, por possuir apenas um cromossomo X 
(XhY), que quando o mesmo sofre uma mutação genética, os sintomas da doença já se manifestam. Já o sexo feminino possui dois cromossomos $\mathrm{X}(\mathrm{XhX})$, sendo necessário que ambos tenham sofrido mutações genéticas para manifestar a doença, já que, apenas um cromossomo X sem alteração genética é capaz de produzir informações necessárias para a produção de fatores de coagulação.

Os resultados encontrados nesse estudo apresentam semelhança com as informações prestadas pelo Departamento de Atenção Especializada sobre o perfil das coagulopatias hereditárias no Brasil (Brasil. Ministerio Da Saúde, 2015) a maior prevalência de hemofilias envolve indivíduos na faixa etária entre 20 a 29 anos no Brasil, fato encontrado neste estudo.

Em geral a hemofilia é detectada em crianças logo no primeiro ano de vida, com manifestações clinicas como manchas rochas pelo corpo conhecidas como equimoses, e tornam-se mais frequentes quando a criança começa a andar e a cair. $\mathrm{O}$ indivíduo com patologia quando se machuca, não sangra mais rápido do que outro sem hemofilia, porém, sangra durante um período maior, podendo continuar a sangrar vários dias depois de um ferimento ou de uma cirurgia (Pereira, 2010).

Consoante o Ministério Da Saúde (2014), encontra-se maior prevalência em indivíduos com faixa etária entre 20 a 29 anos no Brasil, fato encontrado neste estudo. A Hemofilia B de acordo com Rosendaal, Smit, Briët, 1991 é cerca de quatro vezes menos prevalente que a Hemofilia A, devido à ausência do fator de coagulação IX. As manifestações clinicas da Hemofilia A e B são semelhantes, caracterizadas por episódios de sangramento (Beltrame, 2005).

Para que o paciente tenha maior segurança no tratamento das coagulopatias, segundo Covas \& Bordin (2007), a forma mais segura de reposição destes fatores é o uso de concentrados liofilizados específicos. O ministério da saúde determina que, para o tratamento de substituição, deve-se sempre empregar os concentrados comerciais de fatores VIII ou fator IX, sendo possível o uso do crioprecipitado para a reposição de fator VIII e do plasma fresco congelado para a reposição do fator IX, somente quando há a falta dos concentrados comerciais.

O tratamento profilático, a imunotolerância e o tratamento sob demanda devem acontecer na residência do paciente, por meio da terapia domiciliar. Dessa maneira, o paciente não precisa sempre deslocar-se ao Centro de Tratamento de Hemofilia (CTH) quando precisar receber a infusão, o que dá mais autonomia e independência à pessoa com hemofilia e seus familiares (Silva, et al., 2020)

O Manual de Hemofilia (2015) estabelece que todo paciente tem por direito levar uma determinada quantidade de doses domiciliares (DD) para sua residência especialmente pacientes que moram em locais distantes mas isso irá depender da gravidade da hemofilia, mas qualquer paciente pode aderir ao tratamento domiciliar, para isso é necessário apenas que o paciente esteja cadastrado em um centro de tratamento, e realize todos os exames indicados pelo hematologista, e posteriormente seja capacitado para realizar a infusão intravenosa. Esta capacitação é oferecida ao paciente e/ou responsável no próprio centro de tratamento de hemofilia, oferecendo ao paciente um treinamento teórico e prático. No treinamento prático, os pacientes e familiares são treinados para o preparo e a administração do fator.

O paciente hemofílico A grave ou com sintomas grave tem direito a levar até 12 doses de fator de coagulação para seu domicílio para uso nestas circunstâncias. O paciente com hemofilia B grave ou com sintomas de grave tem direito a levar até nove doses para casa. Se o paciente reside longe da unidade onde é realizado o tratamento, é indicado que sejam liberadas mais doses domiciliares. Os kits para tratamento das hemofilias disponibilizados pelo SUS são da Baxter, neste vem presente todo suporte necessário para que o paciente possa fazer a administração do fator (água, fator, baxjet, seringa, algodão e curativos), (Silingowschi, et al., 2015)

Visto que os fatores de reposição são estranhos ao corpo, há sempre o risco do sistema imunológico identificá-los e rejeitá-los. Essa rejeição é realizada pelos anticorpos. Normalmente estes ajudam a proteger o organismo de substâncias nocivas, mas neste caso, neutralizam os fatores VIII ou IX que foram adicionados ao sangue, impedindo assim a sua ação e interrompimento do sangramento. (Chaves, et al., 2009). 
Algumas alternativas terapêuticas foram desenvolvidas para diminuir as complicações que surgem na presença de inibidores, por exemplo, o uso de concentrado de complexo protrombínico (PCCs) ou concentrado de complexo protrombínico ativado (APCCs) podendo assim estimular a formação de um coágulo e cessar a hemorragia, superando o requerimento do FVIII. Porém, este tipo de terapia apresenta algumas limitações, podendo causar excesso de coagulação. Além disso, estes produtos contêm pequenas quantidades de FVIII e maiores quantidades de FIX, podendo assim estimular uma nova inibição tanto para o FVIII na hemofilia A quanto para o FIX na hemofilia B. (Calefi, et al., 2009)

O aumento gradativo dos valores citados a cima, é consequência da implantação de políticas públicas e investimentos do governo federal na obtenção de novas doses de fatores de coagulação, para garantir que o indivíduo com coagulopatia tenha acesso ao tratamento. Segundo índices do portal Brasil, o Ministério da Saúde iria gastar cerca de 880 milhões de reais com a assistência aos hemofílicos, sendo cerca de três vezes maior que em 2008, que totalizou 234 milhões de reais (Brasil, Ministério Da Saúde. 2014).

\section{Considerações Finais}

A Hemofilia do tipo A e B é uma enfermidade que tem sido muito pesquisada no decorrer dos últimos anos, entretanto abarca diversas situações complexas que carecem de ser entendidos. As técnicas laboratoriais usadas para o diagnóstico e monitoramento da enfermidade, proporciona informações reduzidas devido as complexidades de quantificação de níveis baixos de anticorpos e distinção da hemofilia com as demais coagulopatias.

Diante dos resultados desta pesquisa foi possível concluir que esse estudo é semelhante com as informações encontradas nas plataformas cientificas que demonstram maior índice da hemofilia em indivíduos do sexo masculino. Em relação a faixa etária dos pacientes identificados com hemofilia A à maior prevalência foi entre 16 a 30 anos. Essa idade é considerada como um desafio a adesão do tratamento devido a não aceitação e a falta de entendimento a respeito das consequências gerada pela doença.

Em relação a localização da moradia a maioria residem na região urbana o que facilita a ida até o hemocentro para receber a infusão dos fatores, tendo em vista que a distância dificulta o acesso ao tratamento. Dentre os fatores utilizados nos tratamentos das coagulopatias, o maior índice de uso foi o fator VIII recombinante, em relação a quantidade de (UI) dispensados pelo estabelecimento de saúde totalizou 12.940.500 durante o período de 2018 à 2019, destacando os meses de agosto de 2018 e junho de 2019 .

Espera-se que este estudo possa sensibilizar os gestores na área de saúde afim de que os atendimentos ambulatoriais aos hemofílicos não sofram solução de continuidade devido a política de saúde instalada no território brasileiro afim de que os pacientes recebam apoio da equipe multidisciplinar, realização gratuita dos exames laboratoriais, medicações sem ônus para os portadores da doença e monitoramento constante afim de que possam ter qualidade de vida.

Que esta reflexão possa produzir impactos positivos para o desenvolvimento de novos estudos, no que diz respeito à segurança e qualidade da assistência e tratamento das coagulopatias hereditárias.

\section{Referências}

Alcântara, A. L. M. (2019). Hemofilia: fisiopatologia e tratamentos. 2019.Trabalho de Conclusão de Curso (Graduação em Biomedicina) - Faculdade de Ciências da Educação e Saúde, Centro Universitário de Brasília.

Beltrame, L. G. N. (2005). Hemofilia: vida ativa. CREF/DF.

Bogliolo, L., Brasileiro, F. G., \& Barbosa, A. J. A. (2013). Bogliolo Patologia Geral. Guanabara Koogan.

Brasil, Ministério da Saúde. (2008). Hemofilia congênita e inibidor: manual de diagnóstico e tratamento de eventos hemorrágicos. Editora do Ministério da Saúde, Secretaria de Atenção à Saúde, Departamento de Atenção Especializada. 
Brasil, Ministério da Saúde. (2015). Manual de reabilitação na hemofilia / Ministério da Saúde. Secretaria de Atenção à Saúde. Departamento de Atenção Especializada. Coordenação-Geral de Sangue e Hemoderivados. 09.

Brasil, Ministério da Saúde. (2015). Manual de Hemofilia. Secretaria de Atenção à Saúde. Departamento de Atenção Especializada e Temática.

Brasil. Ministério da Saúde. (2016). Secretaria de Atenção à Saúde. Departamento de Atenção Especializada e Temática. Perfil das Coagulopatias Hereditárias.

Brasil. Ministério da Saúde, (2015). Secretaria de Atenção à Saúde. Departamento de Atenção Especializada e Temática. Coordenação-Geral de Sangue e Hemoderivados. Ministério da Saúde, 57.

Brasil. Ministério da Saúde. (2015). Secretaria de Atenção à Saúde. Departamento de Atenção Especializada e Temática. Perfil das coagulopatias hereditárias no Brasil.

Calefi, M. P. S. S. (2019). O tratamento da hemofilia: acesso e exclusão na perspectiva da bioética crítica. Dissertação de mestrado em Bioética - Universidade de Brasília.

Eickhoff, H. H., Koch, W., Raderschadt, G. \& Brackmann, H. H. (1997). Arthroscopy for chronic hemophilic synovitis of the knee. Clinical orthopaedics and related research. $343,58-62$.

Evatt, B. (2005). World federation of hemophilia guide to developing a national patient registry. Montreal: World Federation of Hemophilia.

Croteau, S. E. (2018). Evolving Complexity in Hemophilia Management. Pediatr Clin North Am. 65(3), 407-425.

Federação Brasileira De Hemofilia. (2008). Convivendo com a Hemofilia. (2a ed.).

Brasil, Ministério da Saúde. (2015). Convivendo com a Hemofilia: manual de bolso, Federação Brasileira De Hemofilia.

Ferreira, A. A., Leite, I. C. G., Bustamante, M. T., \& Guerra, M. R. (2014). Hemophilia A in Brazil epidemiology and treatment developments. J Blood Med. 5 , $175-84$

Friedman, K. D. \& Rodgers, G. M. (2008). Inherited Coagulation Disorders. In: GREER, J.P. et al. Wintrobe's Clinical Hematology. 12th ed. Philadelphia: Lippincott Williams \& Wilkins. 2, 1379-1424.

Junqueira. P. C., Hamerschlak, N. \& Rosenblit, J. (2009). Hemoterapia clínica. Roca.

Kasper, C. K. (2009). Principles of clotting factor therapy in hemophilia. 15(6),1181-1186.

Lichtman, M. A., Beutler, E., \& Kipps, T. (2005). Manual de hematologia de Williams.

Lima, M. P. R. (2013). Hemofilias A e B.Recife: Faculdade de Boa Viagem.

Lorenzi, T. F. (2011). Manual de hematologia: propedêutica e clínica. Rio de Janeiro: Guanabara Koogan. 4, 537-546.

Mcpherson, R. A. \& Pincus, M. R. Diagnósticos clínicos e tratamento por métodos laboratoriais de Henry.ed. 21.

Ministério Da Saúde, (2016). Brasil atinge meta da OMS e amplia assistência aos hemofílicos. Portal Brasil: <http://www.brasil.gov.br/saude/2015/08/brasilatinge-meta-da-oms-e-amplia-assistencia-aos-hemofilicos>.

Mulder, K. \& Linnás, A. (2004). The target joint. Haemophilia. 10(4), 152-156.

Neves, T. M. P., et al. (2021). A atuação do enfermeiro na consulta de enfermagem ao paciente hematológico: um relato de experiência. Research, Society and Development. 10(4), e15810413916.

Pasquini, R., Zago, M. A. \& Falcão, R. P. (2004). Hematologia: fundamentos e prática. Atheneu.

Pereira, A. (2010). Aspectos sociais da vivência com a hemofilia.

Peyvandi, F., et al. (2006). Genetic diagnosis of haemophilia and other inherited bleeding disorders. Journal of the world Federation of Hemophilia. 12(3), 829.

Pio, S. F., et al. (2009). As bases moleculares da hemofilia A. Revista da Associação Médica Brasileira. 55(2), $213-219$.

Roberts, H. R., Escobar, M. A. \& White, G. C. (2006). Hemophilia A and Hemophilia B. In: LICHTMAN, M.A. Williams Hematology. (7a ed.), McGraw Hillp.1867-8.

Rodrigues, A. (2005). Hemofilia: origem, transmissão e terapia gênica.

Rodrigues, L. M. L, et al. (2018). Avaliação comparativa entre os novos métodos e os métodos tradicionais de diagnósticos laboratoriais para as hemofilias: revisão integrativa. Rev. RBAC, 2(50), 111-117.

Rosendaal, F. R., Smit C. \& Briet, E. (1991). Hemophilia treatment in historical perspective: a review of medical and social developments. Ann Hematol. 62 , 515.

Santos, M. A., et al. (2020). Reflexões sobre o bullying com hemofílicos nas escolas. Research, Society and Development. 9(8), e377985476 
Research, Society and Development, v. 10, n. 9, e15510917813, 2021

(CC BY 4.0) | ISSN 2525-3409 | DOI: http://dx.doi.org/10.33448/rsd-v10i9.17813

Santos, P. C. J. L., Silva, A. M. \& Neto, M. R. (2013). Hematologia Métodos e Interpretação. Roca. $67,363$.

Silingowschi, E.T.M. (2015). Implantação dos protocolos de profilaxias e imunotolêrancia para a hemofilia no Estado do Tocantins.

Silva, J. C. L., Dos Santos, G. K. \& De Sousa, M. N. A. (2020). Qualidade De Vida Dos Hemofílicos: Análise Pós Implantação Do Hemonúcleo E Distribuíção De Fatores Da Coagulação. Ciência \& Desenvolvimento-Revista Eletrônica Da Fainor. 13(1).

Silva, P. H., \& Hashimoto. Y. (2006). Coagulação: Visão Laboratorial da Hemostasia Primária e Secundária. Revinter Ltda. 30-5.

Verrastro, T., Lorenzi, T. F., \& Wendel, S. (2005). Hematologia e Hemoterapia: Fundamentos de morfologia, fisiologia, patologia e clínica. Atheneu. $214-11$.

Vrabic, A. C. A., Ribeiro, C. A., Ohara, C. V. D. S. \& Borba, R. (2012). Difficulties in facing alone the demands of treatment: experiences of the adolescent hemophiliac. Acta Paulista de Enfermagem. 25(2), 204-210.

Werner, E. J, et al. (1993). Prevalence of von Willebrand disease in children: a multiethnic study. The Journal of pediatrics. 123(6), 893-898.

Whitlock, J. \& A. Gaynon, P.S. (1999). Acute lymphocytic leukemia. Wintrobe's Clinical Hematology. 2, $2241-71$. 